\title{
Complex case of methicillin resistant Staphylococcus aureus occipital osteomyelitis and subdural empyema: vancomycin or linezolid?
}

\author{
Dalvir Gill $^{*}$, Natasha Sheikh ${ }^{2}$ and Waleed Javaid ${ }^{3}$ \\ ${ }^{1}$ Department of Internal Medicine, SUNY Upstate Medical University, 750 East Adams Street, Syracuse, New Jersey, USA \\ ${ }^{2}$ Department of Internal Medicine, Ross University School of Medicine, New Jersey, USA, \\ ${ }^{3}$ Department of Infectious Disease, SUNY Upstate Medical University, 750 East Adams Street, Syracuse, New Jersey, 13210, USA
}

Methicillin-resistant staphylococcus aureus (MRSA) is typically considered a nosocomial pathogen, however it has now emerged to cause infections in the community. We report a case of MRSA bacteremia with subdural empyema and occipital osteomyelitis in a female with a history of significant intravenous drug use (IVDU). There are very few reports on MRSA bacteremia leading to problems with central nervous system (CNS) involvement.

27-year-old female with past medical history significant for IVDU presented to emergency department with neck pain, and occipital headache for 1 week. She admitted to recent heroin use. Her vitals were significant for, blood pressure of $102 / 67 \mathrm{~mm} \mathrm{Hg}$, pulse of 116 beats per minute, temperature $36.9^{\circ} \mathrm{C}$ (oral), respiratory rate of 19 breaths per minute, oxygen saturation of $96 \%$ on room air. Physical exam was remarkable for left shoulder hematoma and track marks bilaterally on arms. WBC count was with normal range. Blood cultures grew MRSA, susceptible to vancomycin. Transesophageal echocardiogram showed $6.9 \mathrm{~mm}$ tricuspid valve vegetation. MRI brain showed left subdural empyema (Figure 1) and associated osteomyelitis within the overlying occipital bone (Figure 2). MRI head showed left transverse sinus thrombosis extending superiorly into an occipital lobe cortical vein with evidence of collateralization of flow within the overlying occipital bone (Figure 3). Neurosurgery did not recommend any surgical intervention. Follow up head MRI showed decrease in the size of the subdural empyema. Patient completed 6-week course of vancomycin and her symptoms improved.

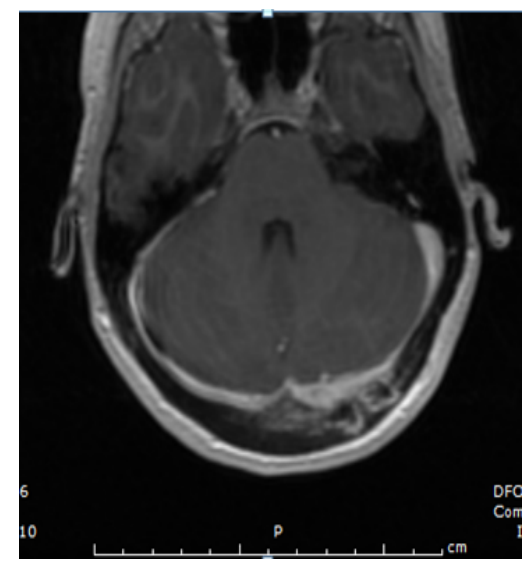

Figure 1. MRI brain showing left subdural empyema.
The patient in our case report developed bacteremia from MRSA due to significant IVDU and it had disseminated into the central nervous system. Treating CNS disease due to MRSA is challenging because of few therapeutic options, and difficulty in attaining therapeutic concentration of the drug in cerebrospinal fluid (CSF). Vancomycin is the main treatment, however in some patient's vancomycin therapy fails because of its poor CSF penetration [1]. There are some cases where linezolid has been successful, due to its greater CSF penetration [2,3]. Even though there are many CNS disease occurring from MRSA, it is rare to find CNS disease development from community acquired MRSA. Hence, clinicians should have a high index of suspicion for subdural empyema in IVDUs who present with headaches and neck pain. Recognition and treatment of subdural empyema as a complication of bacteremia in IVDU is very important due to its complication rate. As evident in this case, thrombosis of the venous sinus can lead to venous occlusion and sometimes even brain infarction. Surgical and/or medical treatment should not be delayed.

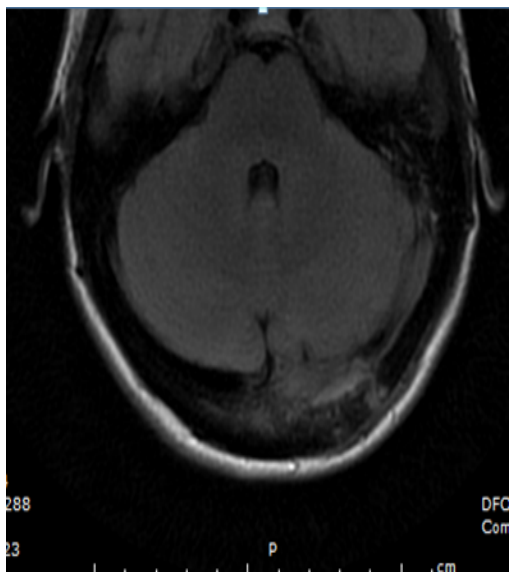

Figure 2. MRI brain showing osteomyelitis within the overlying occipital bone.

Correspondence to: Dalvir Gill, Department of Internal Medicine, SUNY Upstate Medical University, 750 East Adams Street, Syracuse, New Jersey, USA, E-mail: gillda@upstate.edu

Received: January 04, 2017; Accepted: January 30, 2017; Published: February 02,2017 
Gill D (2017) Complex case of methicillin resistant Staphylococcus aureus occipital osteomyelitis and subdural empyema: vancomycin or linezolid?

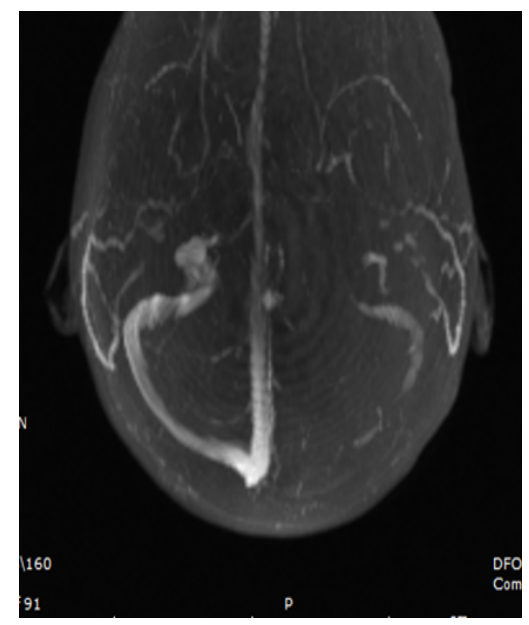

\section{References}

1. Kessler AT, Kourtis AP (2007) Treatment of meningitis caused by methicillinresistant Staphylococcus aureus with linezolid. Infection 35: 271-274.

2. Saito N, Aoki K, Sakurai T (2010) Linezolid treatment for intracranial abscesses caused by methicillin-resistant Staphylococcus aureus - two case reports. Neurol Med Chir (Tokyo) 50: 515-517.

3. Naesens R, Ronsyn M, Druwé P (2009) Central nervous system invasion by communityacquired methicillin-resistant Staphylococcus aureus. J Med Microbiol 58: 1247-1251.

Figure 3. MRI head showing left transverse sinus thrombosis, extending superiorly into an occipital lobe cortical vein with evidence of collateralization of flow within the overlying occipital bone.

Copyright: (C2017 Gill D. This is an open-access article distributed under the terms of the Creative Commons Attribution License, which permits unrestricted use, distribution, and reproduction in any medium, provided the original author and source are credited. 